\title{
Identifying acetylated lignin units in non-wood fibers using pyrolysis-gas chromatography/mass spectrometry
}

\author{
José C. del Río ${ }^{1 *}$, Ana Gutiérrez ${ }^{1}$ and Ángel T. Martínez ${ }^{2}$ \\ ${ }^{1}$ Instituto de Recursos Naturales y Agrobiología de Sevilla, CSIC, P.O. Box 1052, 41080 Seville, Spain \\ ${ }^{2}$ Centro de Investigaciones Biológicas, CSIC, Ramiro de Maeztu 9, 28040 Madrid, Spain \\ Received 2 March 2004; Revised 23 March 2004; Accepted 23 March 2004
}

\begin{abstract}
A series of non-wood plant fibers, namely kenaf, jute, sisal and abaca, have been analyzed upon pyrolysis-gas chromatography/mass spectrometry (Py-GC/MS) of the whole material. The pyrolysis products mainly arise from the carbohydrate and lignin moieties of the fibers. The lignin-derived phenols belonged to the $p$-hydroxyphenylpropanoid $(\mathrm{H})$, guaiacylpropanoid $(\mathrm{G})$ and syringylpropanoid (S) structures, and showed a high S/G ratio of between 2.0 and 5.4, the highest corresponding to kenaf. Among the lignin-derived phenols released, small amounts of sinapyl and coniferyl acetates (in both cis- and trans-forms) were identified for the first time upon Py-GC/MS of lignocellulosic materials. Acetylation of the sinapyl and coniferyl alcohols was at the $\gamma$-position of the side chain. The release of these alcohols derived from intact acetylated lignin units upon pyrolysis seems to indicate that the native lignin in the fibers selected for this study is at least partially acetylated. Sinapyl (and coniferyl) acetates have recently been suggested to be authentic lignin precursors involved in the polymerization of lignin along with the normal sinapyl and coniferyl alcohols. Py-GC/MS will offer a convenient and rapid tool for analyzing naturally acetylated lignins, as well as to screen plant materials for the presence of acetylated units in lignin. Copyright (C) 2004 John Wiley \& Sons, Ltd.
\end{abstract}

Three phenolic monolignols, namely p-hydroxycinnamyl, coniferyl and sinapyl alcohols, are considered as the natural precursors of lignin in vascular plants. ${ }^{1,2}$ The dehydrogenative polymerization of these monolignols initiated by plant oxidoreductases (peroxidases and/or laccases) results in a very complex and recalcitrant three-dimensional macromolecule with different unit types and inter-unit linkages, whose chemical structure is far from being completely established. It has been known for many years that some lignins are naturally acylated but the details and mechanisms are to be investigated. ${ }^{3}$ Grass lignins are partially $p$ coumaroylated, ${ }^{4,5}$ and some hardwood lignins are $p$ hydroxybenzoylated. ${ }^{6,7}$ Acetates have also been reported to occur in some hardwood and non-wood lignins; $;^{8-10}$ however, studies on natural lignin acetates are still scarce.

Information on natural acetylation of lignin may have been biased due to restrictions of the analytical procedures used for its isolation and/or characterization. Natural acetates present in lignin might have been hydrolyzed and removed when using traditional isolation methods (such as alkaline extraction often applied to non-wood lignins) and degradative procedures for chemical characterization (such as nitrobenzene oxidation, $\mathrm{CuO}$ oxidation or thioacidolysis). Indeed, for spectroscopic analysis, e.g. using nuclear

*Correspondence to: J. C. del Río, IRNAS-CSIC, P.O. Box 1052, 41080 Seville, Spain.

E-mail: delrio@irnase.csic.es

Contract/grant sponsor: Spanish Ministerio de Ciencia y Tecnología; contract/grant number: AGL2002-00393. magnetic resonance (NMR), lignin is frequently acetylated for improved spectral properties and, therefore, native acetates cannot be identified.

Recently, a new degradation procedure (the so-called derivatization followed by reductive cleavage, DFRC) has been developed for the analysis of lignin. DFRC cleaves $\alpha$ and $\beta$-ether linkages but leaves $\gamma$-esters intact, and thus it is appropriate for the study of acylated lignin. ${ }^{11}$ This method also uses acetate-containing reagents, but, with appropriate modification avoiding the use of these reagents, it has been possible to obtain significant information and clues about the presence of acetates in lignin. ${ }^{10}$ Hence, it has been shown with DFRC that kenaf bast fiber lignin is extensively acetylated and that acetylation occurs almost entirely at the $\gamma$-position of syringyl units, confirming previous NMR data. ${ }^{9}$ It was also shown that lignin in aspen is slightly $\gamma$-acetylated. ${ }^{10}$

Pyrolysis coupled to gas chromatography/mass spectrometry (Py-GC/MS) is also known to be a powerful analytical tool for the characterization of lignin. Lignin is thermally degraded to produce a mixture of relatively simple phenols, which result from cleavage of ether and certain C-C interunit linkages. ${ }^{12-15}$ The released methoxylated phenols retain the substitution patterns of the different lignin monomers, and it is thus possible to identify components from the $p$ hydroxyphenylpropanoid $(\mathrm{H})$, guaiacylpropanoid $(\mathrm{G})$ and syringylpropanoid (S) lignin units, in which the lignin propanoid side chain has been split off completely, shortened to one or two carbons, or fully conserved. New double bonds are also created in the side chains through pyrolytic 
dehydrogenation. Whereas sinapyl and coniferyl alcohols are usually released in the Py-GC/MS of S and G lignins, their corresponding $\gamma$-acetylated counterparts have never been detected in previous studies. ${ }^{12-15}$ In the present work, we have found intact acetylated lignin units (sinapyl and coniferyl acetates) in kenaf and other non-wood plant fibers (jute, sisal, abaca) after Py-GC/MS. To our knowledge, this is the first time that these compounds have been detected upon Py-GC/MS, which will make this technique valuable for the screening of naturally acetylated lignins.

\section{EXPERIMENTAL}

\section{Non-wood fibers}

The non-woody plant materials used for this study consisted of bast fibers obtained from the stalk phloem layer, and leaf fibers. Among the bast fibers, jute (Corchorus capsularis) and kenaf (Hibiscus cannabinus) were selected, while sisal (Agave sisalana) and abaca (Musa textilis) were selected among the leaf fibers. Jute and kenaf samples had been previously subjected to a retting process, which is a biological treatment that releases fiber bundles by dissolving pectic substances. Bast and leaf fibers were finely ground to sawdust using a knife mill (Janke and Kunkel, Analysenmühle) before analysis.

\section{Py-GC/MS}

The pyrolyses were performed with a Curie-point flash pyrolyzer coupled to a Varian Saturn 2000 GC/MS system using a $30 \mathrm{~m} \times 0.25 \mathrm{~mm}$ DB-5 column (film thickness $0.25 \mu \mathrm{m}$ ).
Approximately $100 \mu \mathrm{g}$ of finely divided sample were deposited on a ferromagnetic wire then inserted into the glass liner and placed immediately in the pyrolyzer. The pyrolysis was carried out in the glass liner at $610^{\circ} \mathrm{C}$ for $4 \mathrm{~s}$. The chromatograph was programmed from $40^{\circ} \mathrm{C}(1 \mathrm{~min})$ to $300^{\circ} \mathrm{C}$ at a rate of $6^{\circ} \mathrm{C} / \mathrm{min}$. The final temperature was held for $20 \mathrm{~min}$. The injector temperature was kept at $280^{\circ} \mathrm{C}$ while the GC/MS interface was kept at $300^{\circ} \mathrm{C}$. The compounds were identified by comparison with those reported in the literature, ${ }^{14,15}$ and in the Wiley and NIST computer libraries.

\section{RESULTS AND DISCUSSION}

The non-wood fibers selected for this study (kenaf, jute, sisal and abaca) were analyzed upon Py-GC/MS. The pyrogram of a selected fiber sample, sisal (Agave sisalana), is shown in Fig. 1. The pyrolysis products identified arise from carbohydrate and phenylpropanoid compounds (lignin and $p$ hydroxycinnamic acids). The lignin-derived phenols released belong to the $p$-hydroxyphenylpropanoid $(\mathrm{H})$, guaiacylpropanoid $(\mathrm{G})$ and syringylpropanoid (S) units. Major lignin-derived compounds were syringol (35), vanillin (39), 4-methylsyringol (41), trans-isoeugenol (42), 4-vinylsyringol (48), 4-allyl-2,6-dimethoxyphenol (50), cis-4-propenylsyringol (52), syringaldehyde (53), trans-4-propenylsyringol (56), syringylacetone (60), trans-sinapaldehyde (67) and trans-sinapyl alcohol (68). Products arising from pyrolysis of carbohydrates were also present in all the pyrograms, levoglucosane (44) being the most prominent. Relative molar

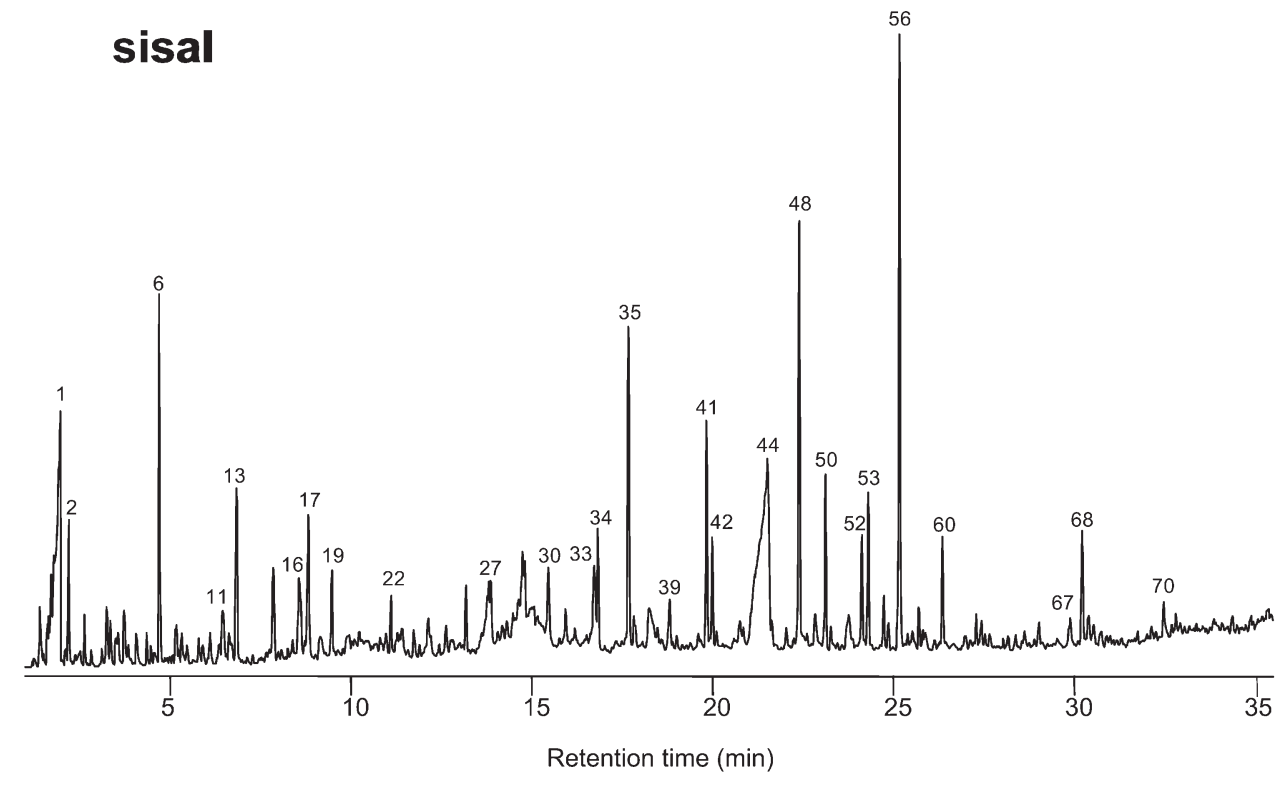

Figure 1. Py-GC/MS of a selected non-wood fiber (sisal). Identification of main peaks (relative molar area $\geq 1 \%$ ): 1, hydroxyacetaldehyde; 2, 3-hydroxypropanal; 6, 2-furaldehyde; 11, 5(H)-furan-2-one; 13, 2,3-dihydro-5-methylfuran-2-one; 16, 5,6-dihydropyran-2,5-dione; 17, 4-hydroxy-5,6-dihydro-(2H)-pyran-2-one; 19, 2-hydroxy-3-methyl-2-cyclopenten-1-one; 22, guaiacol; 27, catechol; 30, 3-methoxycatechol; 33, 1,4-dideoxy-D-glycerohex-1enopyrenon-3-ulone; 34, 4-vinylguaiacol; 35, syringol; 39, vanillin; 41, 4-methylsyringol; 42, trans-isoeugenol; 44, levoglucosane; 48, 4-vinylsyringol; 50, 4-allyl-2,6-dimethoxyphenol; 52, cis-4-propenylsyringol; 53, syringaldehyde; 56, trans-4-propenylsyringol; 60, syringylacetone; 67, trans-sinapaldehyde; 68, trans-sinapyl alcohol; and 70, trans-sinapyl acetate. 
Table 1. Molar abundances of total H-, G- and S-type compounds after Py-GC/MS of non-wood fibers, including p-hydroxycinnamyl acetates, and $S / G$ and phenol/ carbohydrate ratios $^{a}$

\begin{tabular}{lrrrr}
\hline & Kenaf & Jute & Sisal & Abaca \\
\hline$\% \mathrm{H}$ & 1.3 & 2.1 & 1.3 & $63.6^{\mathrm{b}}$ \\
$\% \mathrm{G}$ & 15.4 & 32.2 & 18.7 & 6.4 \\
$\% \mathrm{~S}$ & 83.3 & 65.7 & 80.0 & 30.0 \\
$\mathrm{~S} / \mathrm{G}$ & 5.4 & 2.0 & 4.3 & 4.7 \\
Phenols/carbohydrates & 1.1 & 0.8 & 0.6 & 0.9 \\
cis-Coniferyl acetate & 0.1 & $<0.1$ & 0.2 & $<0.1$ \\
trans-Coniferyl acetate & 0.8 & 0.2 & 0.7 & 0.1 \\
cis-Sinapyl acetate & 0.6 & 0.2 & 0.3 & $<0.1$ \\
trans-Sinapyl acetate & 1.6 & 0.6 & 1.0 & 0.2 \\
\hline
\end{tabular}

${ }^{a}$ Molar areas as percentages of total peak areas of Py-GC/MS compounds.

${ }^{\mathrm{b}}$ Includes high amount of 4-vinylphenol (23.7\%) arising from $p$ coumaric acid. peak areas were calculated for carbohydrate- and ligninderived pyrolysis products. All the selected fibers released high amounts of phenols arising from lignin (and $p$-hydroxycinnamic acids) having a phenol/carbohydrate molar ratio of 0.6-1.1, the highest being for kenaf fiber (Table 1). Indeed, a predominance of S- over G-type compounds was found in all samples, with a S/G ratio between 2.0 and 5.4, the highest value being also for kenaf (Table 1 ).

A detailed analysis of the compounds released after PyGC/MS of the fibers selected here revealed the significant presence of a compound (peak 70) that has not been previously identified among the pyrolysis products of other lignocellulosic materials. ${ }^{12-15}$ The mass spectrum of this compound, shown in Fig. 2(a), indicates that it corresponds to a sinapyl alcohol acetylated at the $\gamma$-position of the side chain. The mass spectrum shows a molecular ion at $\mathrm{m} / \mathrm{z} 252$ and fragments arising from the loss of the acetyl group at [M$\left.\mathrm{CH}_{3} \mathrm{CO}\right]^{+}$and $\left[\mathrm{M}-\mathrm{CH}_{3} \mathrm{COOH}\right]^{+}$at $m / z 209$ and 192, (a)

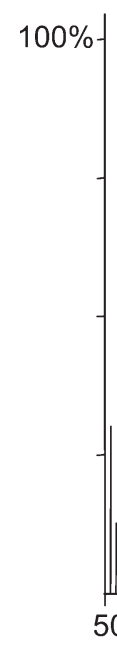

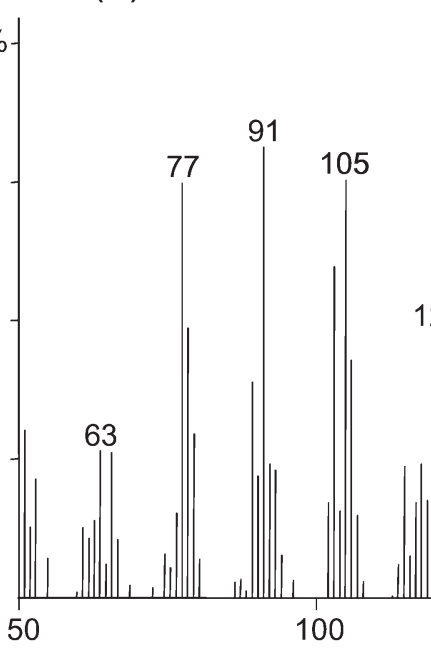

(b)

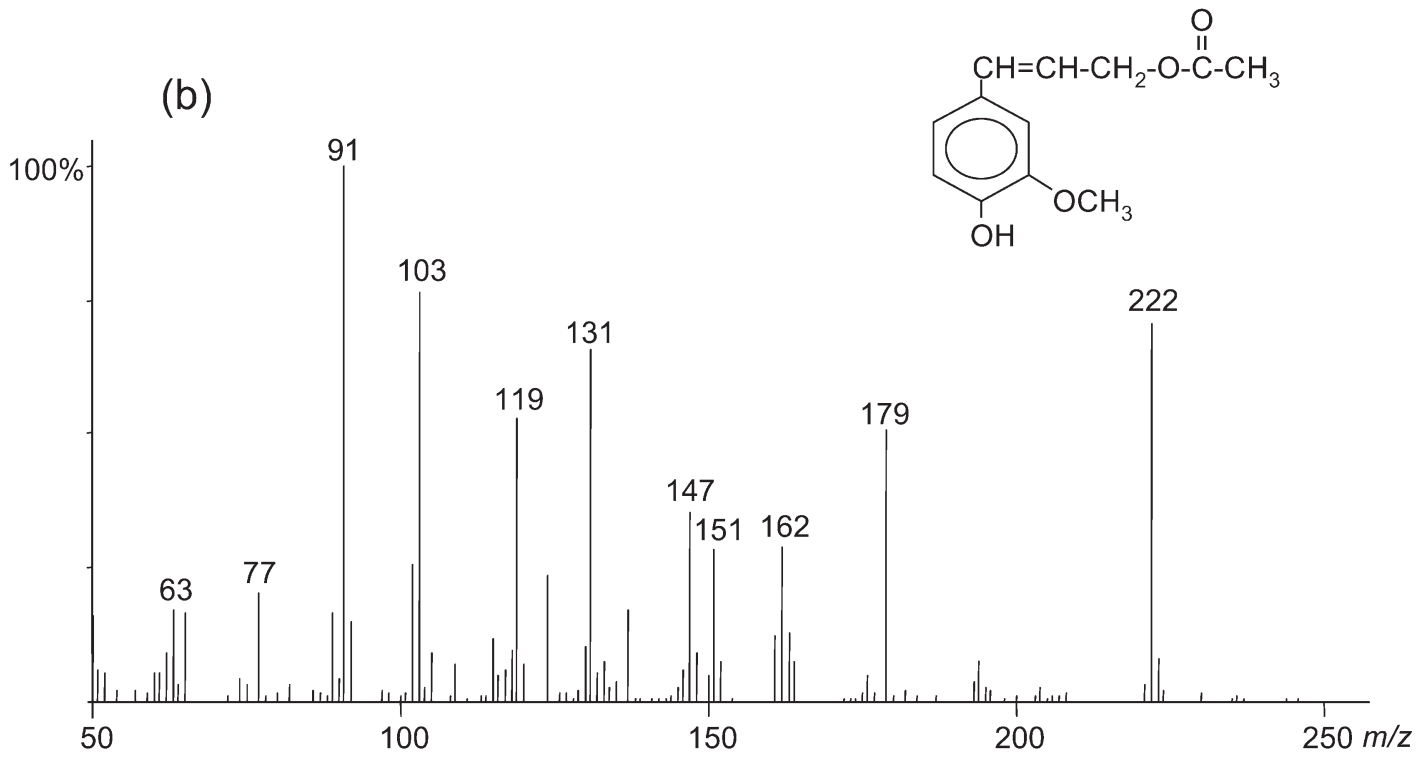

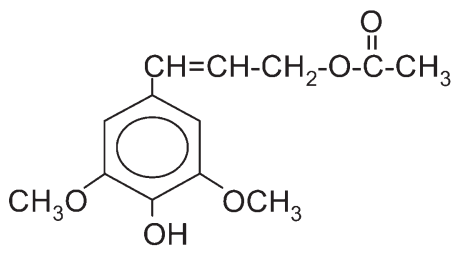

161

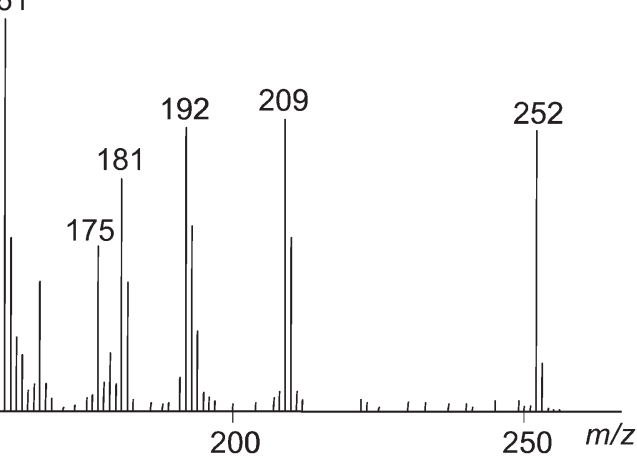

Figure 2. Mass spectra of (a) sinapyl acetate and (b) coniferyl acetate, found after Py-GC/MS of nonwood fibers. 

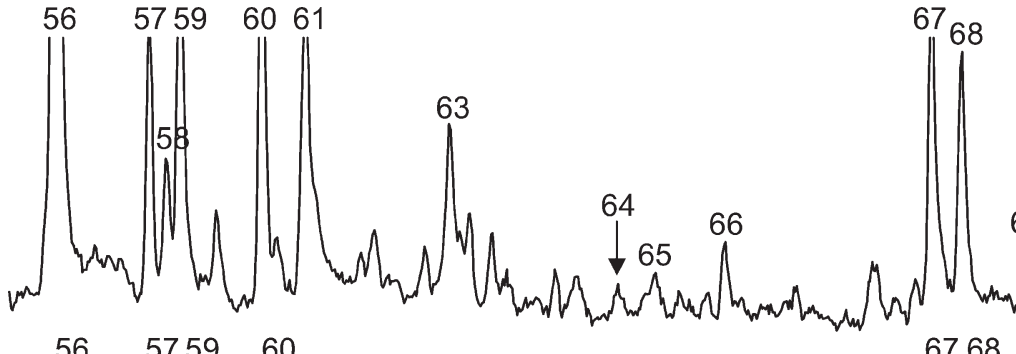

abaca

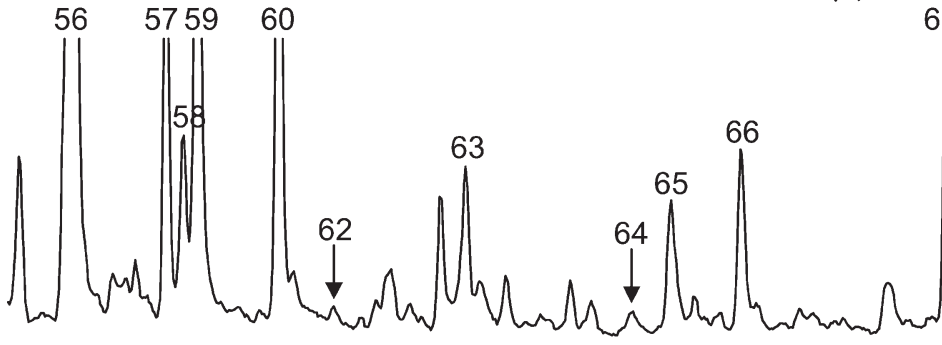

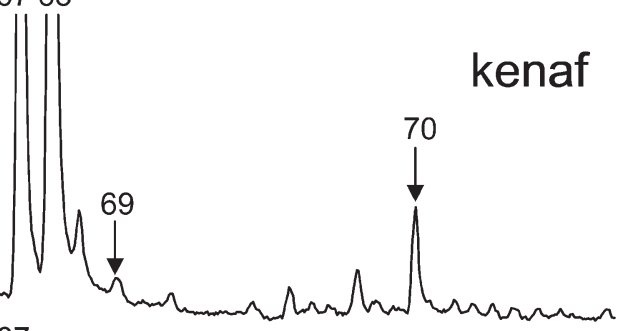

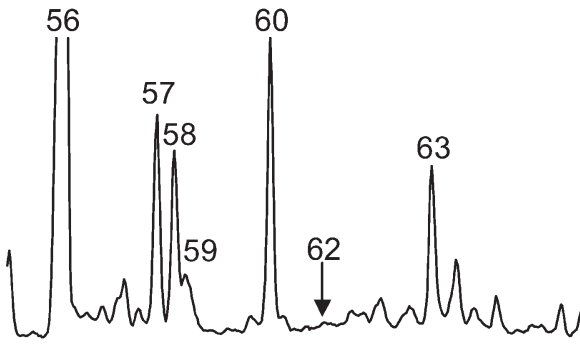

67

jute

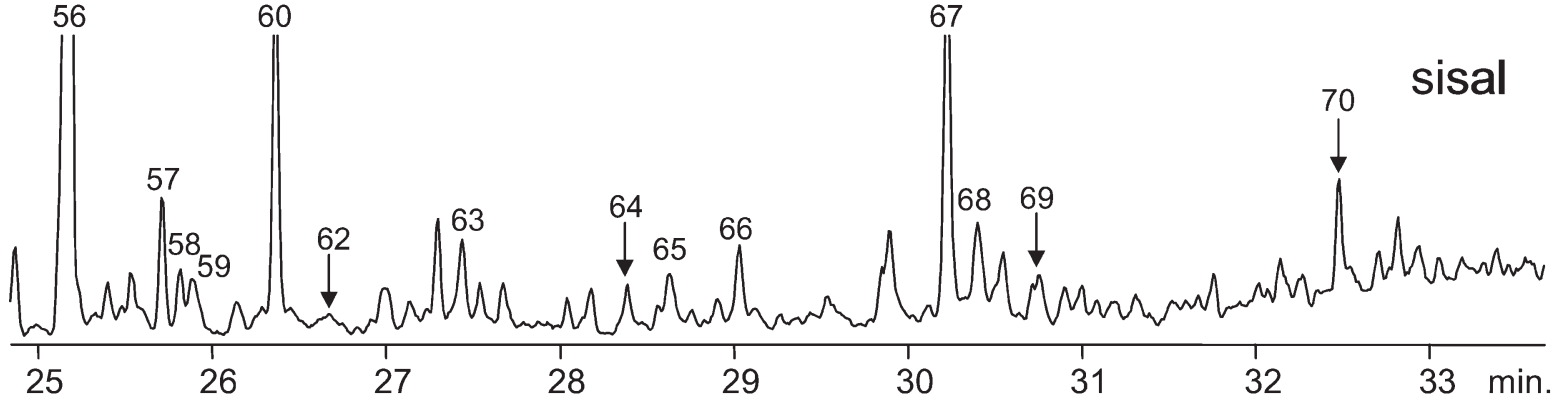

$m / z 222$

64

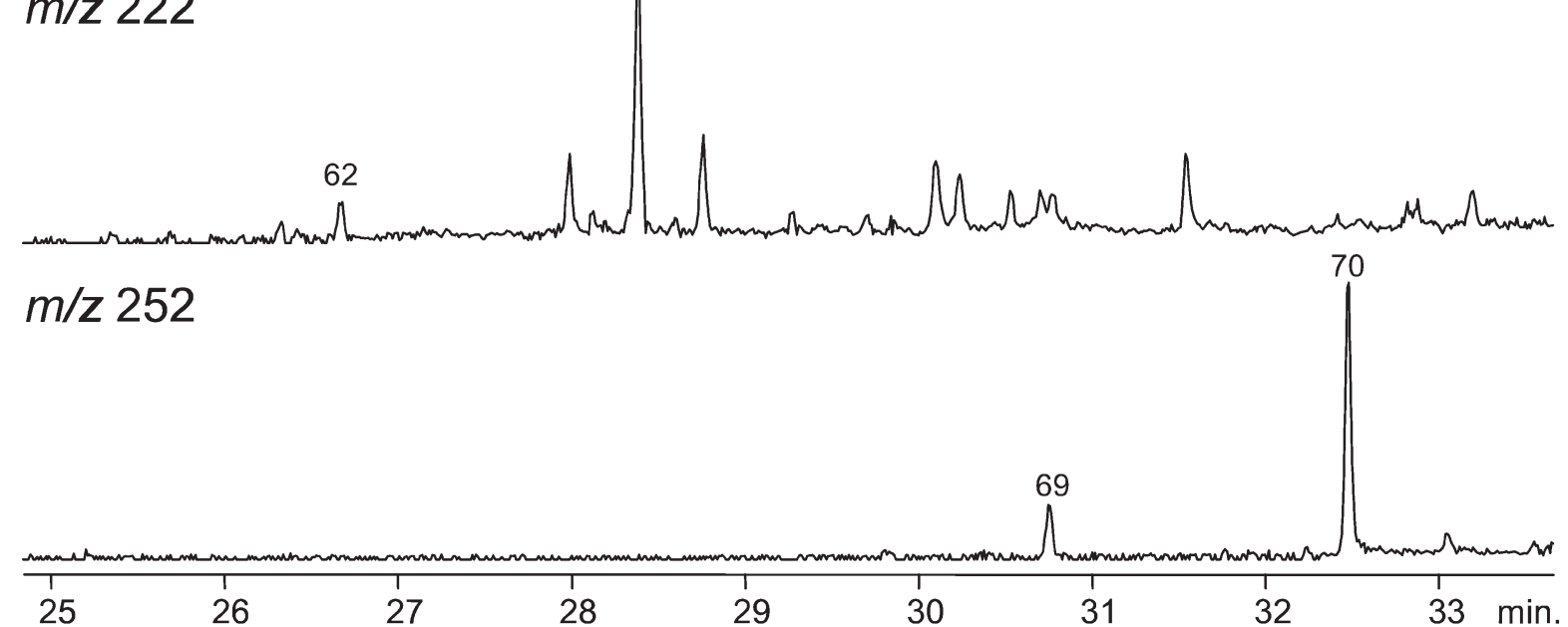

Figure 3. Detection of $p$-hydroxycinnamyl acetates: Detail of total-ion pyrograms of abaca, kenaf, jute and sisal (top), and reconstructed pyrograms showing coniferyl acetate $(\mathrm{m} / \mathrm{z} 222$ ion) and sinapyl acetate peaks $(\mathrm{m} / \mathrm{z} 252$ ion) in a selected sample, sisal (bottom). Peak identification: 56, trans-propenylsyringol; 57, acetosyringone; 58, transconiferaldehyde; 59, trans-coniferyl alcohol; 60, syringylacetone; 61, p-coumaric acid; 62, cis-coniferyl acetate; 63, propiosyringone; 64, trans-coniferyl acetate; 65, dihydrosinapyl alcohol; 66, cis-sinapyl alcohol; 67, transsinapaldehyde; 68, trans-sinapyl alcohol; 69, cis-sinapyl acetate; and 70, trans-sinapyl acetate. 
respectively. From the latter, successive losses of the two methoxyl groups in the aromatic ring give the fragments at $m /$ $z 161$ and 131. The rest of the spectrum is similar to that of sinapyl diacetate after loss of two acetyl groups. ${ }^{11}$ Sinapyl acetate was found in both cis- and trans-forms in all the samples analyzed.

Minor amounts of coniferyl acetate (in cis- and trans-forms) were also detected during Py-GC/MS of kenaf, jute, sisal and abaca. The mass spectrum of this compound, shown in Fig. 2(b), presents a molecular ion at $m / z 222$ and fragments due to the losses of the acetyl group at $\left[\mathrm{M}-\mathrm{CH}_{3} \mathrm{CO}\right]^{+}$and $[\mathrm{M}-$ $\left.\mathrm{CH}_{3} \mathrm{COOH}\right]^{+}$at $m / z 179$ and 162 , respectively. Subsequent loss of the methoxyl group in the aromatic ring gives the fragment at $m / z 131$. The rest of the spectrum is similar to the mass spectrum of the coniferyl diacetate after loss of two acetyl groups. ${ }^{11} p$-Hydroxycinnamyl $(\mathrm{H})$ acetate could only be tentatively identified because of the very low abundance of such potential product.

Figure 3 shows the presence of coniferyl acetates (peaks 62 and 64) and sinapyl acetates (peaks 69 and 70) in the pyrolysates of the different samples analyzed. In all samples, the coniferyl and sinapyl acetates show a predominance of the trans- over the cis-form, as also occurs with the corresponding non-acetylated alcohols. Sinapyl acetates predominate over the respective coniferyl acetates in all samples.

These compounds are unlikely to be artifacts produced during pyrolysis since Py-GC/MS of many other lignins and plant materials lacked these compounds. Indeed, no acetylated reagents were used during the analyses, and the fibers were directly subjected to Py-GC/MS without prior lignin isolation. Moreover, these compounds could not be detected in the alkaline lignins isolated from the same fibers, indicating that acetates units were hydrolyzed and removed during lignin isolation. The presence of acetylated lignin units may have been biased in previous Py-GC/MS studies of lignocellulosic materials owing to the lack of a proper identification or perhaps due to its absence in the materials studied. Although a part of the originally occurring lignin acetate moieties may have been removed during the pyrolysis, coniferyl and sinapyl acetates can still be detected intact among the pyrolysis products.

It has already been shown by NMR that kenaf bast fiber lignin is highly acetylated, and that acetylation occurs almost entirely at the primary $\gamma$-position of the side chain. ${ }^{9}$ By using the DFRC method, it was possible to confirm the NMR results and demonstrate that acetylation in kenaf bast lignin occurs overwhelmingly on syringyl units. ${ }^{10}$ Indeed, it was also possible to show that acetylation occurs at the monolignol stages and that sinapyl acetate becomes a monomer in the polymerization of lignin (along with the sinapyl and coniferyl alcohols) in kenaf and, therefore, it appears to be an authentic lignin precursor. ${ }^{16}$ This is the first time that acetylated lignin units have been reported in sisal, jute and abaca. It is possible that, in these plants, sinapyl acetate is also a lignin precursor, as suggested for the kenaf lignin. ${ }^{16}$

Interestingly, sinapyl and coniferyl acetates have only been found in non-wood fibers characterized for their high S/G ratio. These compounds could not be detected in the pyrolysates of other non-wood fibers analyzed in our laboratories, such as hemp and flax, with low S/G ratios ( 0.8 and 0.3 , respectively). The function of such an acetylated lignin for the plant is not yet known, although it has already been pointed out that this might be a mechanism to achieve syringyl-rich lignins. ${ }^{9}$ However, these compounds could not be detected in the Py-GC/MS of eucalypt wood, a hardwood also characterized by a high S/G ratio. In any case, the fact that acetylated lignin units are present in both monocotyledons (sisal and abaca) and dicotyledons (kenaf and jute) suggests that they might be widespread among syringyl-rich non-wood fibers. Finally, if the presence of acetylated units in lignin is due to participation of sinapyl and coniferyl acetates in biosynthesis as true precursors, as suggested for kenaf, ${ }^{16}$ the traditional concepts of both lignin biosynthesis and structure should be reconsidered. This indicates, in agreement with other authors, ${ }^{17}$ that the lignification process is very flexible, and that a current definition of lignin must include more than the three traditional hydroxycinnamyl alcohols ( $p$-coumaryl, coniferyl and sinapyl alcohols).

\section{CONCLUSIONS}

Py-GC/MS allowed the detection for the first time of sinapyl and coniferyl acetates, which have recently been suggested to be original lignin precursors, in lignocellulosic materials. Acetylated lignin precursors were found in kenaf and, for the first time, in other non-wood fibers such as jute, sisal and abaca. This technique proved to be a powerful and rapid tool for analyzing naturally acetylated lignins and will allow researchers to screen plant materials for the presence of acetylated units in lignin.

\section{Acknowledgements}

This study was supported by the Spanish Ministerio de Ciencia y Tecnología (MCyT) (project AGL2002-00393). A. G. acknowledges a "Ramón y Cajal" contract of the Spanish MCyT. We also thank J. M. Gras and G. Artal (CELESA, Spain) for providing the plant samples.

\section{REFERENCES}

1. Freudenberg K, Neish AC. Constitution and Biosynthesis of Lignin. Springer-Verlag: Berlin, 1968.

2. Higuchi T. Biochemistry and Molecular Biology of Wood. Springer-Verlag: London, 1997.

3. Smith DCC. Nature 1955; 176: 267.

4. Monties B, Lapierre C. Physiol. Veg. 1981; 19: 327.

5. Lu F, Ralph J. J. Agric. Food Chem. 1999; 47: 1988.

6. Nakano J, Ishizu A, Migata N. Tappi 1961; 44: 30.

7. Landucci LL, Deka GC, Roy DN. Holzforschung 1992; 46: 505.

8. Sarkanen KV, Chang H-M, Allan GG. Tappi 1967; 50: 587.

9. Ralph J. I. Nat. Products 1996; 59: 341.

10. Ralph J, Lu F. J. Agric. Food Chem. 1998; 46: 4616.

11. Lu F, Ralph J. J. Agric. Food Chem. 1997; 45: 4655.

12. Saiz-Jiménez C, de Leew JW. Org. Geochem. 1984; 6: 417.

13. Saiz-Jiménez C, de Leew JW. Org. Geochem. 1986; 10: 869.

14. Faix O, Meier D, Fortman I. Holz Roh-Werkst. 1990; 48: 281.

15. Ralph J, Hatfield RD. J. Agric. Food Chem. 1991; 39: 1426.

16. Lu F, Ralph J. Chem. Comm. 2002; 90.

17. Sederoff RD, MacKay J, Ralph J, Hatfield R. Curr. Opin. Plant Biol. 1999; 2: 145. 